\title{
Perturbative complexity of an interacting theory
}

\author{
Wung-Hong Huang $\oplus^{*}$ \\ Department of Physics, National Cheng Kung University, No. 1, University Road, Tainan 701, Taiwan
}

(Received 13 September 2020; accepted 5 February 2021; published 8 March 2021)

\begin{abstract}
We present a systematic method to expand the quantum complexity of interacting theory in a series of coupling constants. The complexity is evaluated by the operator approach in which the transformation matrix between the second quantization operators of the reference state and the target state defines the quantum gate. We start with two coupled oscillators and perturbatively evaluate the geodesic length of the associated group manifold of the gate matrix. Next, we generalize the analysis to $N$ coupled oscillators, which describes the lattice $\lambda \phi^{4}$ theory. Especially, we introduce simple diagrams to represent the perturbative series and construct simple rules to efficiently calculate the complexity. General formulas are obtained for the higher-order complexity of excited states. We present several diagrams to illuminate the properties of complexity and show that the interaction correction to complexity may be positive or negative depending on the magnitude of the reference-state frequency.
\end{abstract}

DOI: 10.1103/PhysRevD.103.065002

\section{INTRODUCTION}

Achieving a better understanding of physics behind a black hole horizon is important if one wants to precisely describe the bulk geometry in terms of the information of boundary conformal field theory (CFT) [1-7]. In the context of the eternal anti-de Sitter Schwarzchild black hole, for instance, a related question concerns the size of a wormhole growing linearly with time; this phenomenon has been conjectured to dual to the growth of "complexity" of the dual CFT [8]. In the complexity = volume (CV) conjecture [8], the complexity is dual to the volume of an extremal codimension-one bulk surface anchored to a time slice of the boundary. In the complexity = action conjecture [9-12], one identifies the complexity with a gravitational action evaluated on the Wheeler-DeWitt patch, anchored also on a time slice of the boundary.

Several efforts were made to provide a definition of the complexity in the field theory [13-17]. The complexity in there is defined as the number of operations $\left\{\mathcal{O}^{I}\right\}$ needed to transform a reference state $\left|\psi_{R}\right\rangle$ to a target state $\left|\psi_{T}\right\rangle$. These operators are also called quantum gates: the more gates one needs, the more complex the target state is. One can define an affine parameter " $s$ " associated with an unitary operator $U(s)$ and use a set of functions $Y^{I}(s)$ to

\footnotetext{
*whhwung@mail.ncku.edu.tw
}

Published by the American Physical Society under the terms of the Creative Commons Attribution 4.0 International license. Further distribution of this work must maintain attribution to the author(s) and the published article's title, journal citation, and DOI. Funded by SCOAP ${ }^{3}$. characterize the quantum circuit. The unitary operation connecting the reference state and target state is

$$
\begin{aligned}
U(s) & =\overrightarrow{\mathcal{P}} e^{\int_{0}^{s} Y_{I}(s) \mathcal{O}_{I}}, \quad\left|\psi_{R}\right\rangle=U(0)\left|\psi_{R}\right\rangle, \\
\left|\psi_{T}\right\rangle & =U(1)\left|\psi_{R}\right\rangle,
\end{aligned}
$$

where $\overrightarrow{\mathcal{P}}$ is a time ordering along $s$. The complexity $\mathcal{C}$ and circuit depth $D[U]$ (cost function) are [13]

$$
\mathcal{C}=\operatorname{Min}_{\{\mathrm{YI}\}} D[U], \quad D[U]=\int_{0}^{1} d s \sum_{I}\left|Y^{I}(s)\right|^{2} .
$$

The above definitions were shown to be consistent with a gravitational computation [13]. The initial studies in field theory considered the Gaussian ground-state wave functions in the reference and target states $[13,15,16]$. The theories studied so far are the free field theory or exponential type wave function in interacting model [17]. The operator approach had also been used in $[14,16]$ to study the complexity of fermion theory.

In our previous paper [18], we adopted the operator approach, in which the transformation matrix between the second quantization operators of reference and target states is regarded as the quantum gate, to evaluate the complexity in free scalar field theory. Since in the operator approach we need not to use the explicit form of the wave function, we can study the complexity in the excited states. ${ }^{1}$ We first

\footnotetext{
${ }^{1}$ Note that the excited-state wave function of harmonic oscillation is not pure exponential form and the wave function approach is hard to work.
} 


$$
\text { (j) } \Rightarrow A(j)^{4}
$$

FIG. 1. The circle element.

$$
\bigsqcup^{\mathrm{i}} \quad \mathrm{j} \Rightarrow 6 A(i)^{2} A(j)^{2}
$$

FIG. 2. The pair element.

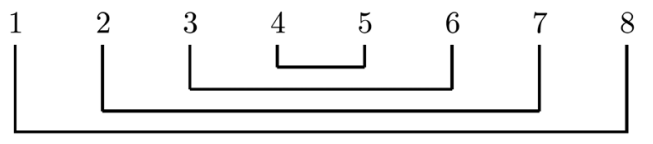

FIG. 3. $N=9$ diagram.

examined the system in which the reference state is two oscillators with same frequency $\omega_{f}$, while the target state is two oscillators with frequency $\omega_{1}$ and $\omega_{2}$. We explicitly calculated the complexity in several excited states and proved that the square of geodesic length in the general state $\left|N_{1}, N_{2}\right\rangle$ is

$D_{\left(N_{1}, N_{2}\right)}^{2}=\left(N_{1}+1\right)\left(\ln \sqrt{\frac{\omega_{1}}{\omega_{f}}}\right)^{2}+\left(N_{2}+1\right)\left(\ln \sqrt{\frac{\omega_{2}}{\omega_{f}}}\right)^{2}$.

The results were furthermore extended to the $N$ coupled harmonic oscillators, which correspond to the lattice version of free scalar field (see Sec. V of [18]).

In this paper, we extend [18] by including interactions to further study the complexity using the operator approach. We present a systematic method to evaluate the complexity of the $\lambda \phi^{4}$ field theory by the perturbation of small coupling constant. An outline of the paper is as follows.

In Sec. II, as in [13], we describe the lattice scalar field as coupled oscillators. In Sec. III, we consider two coupled oscillators and find that, to the $\lambda^{n}$ order, the square distance of the excited state between target and reference states is

$$
\begin{aligned}
D_{\left(N_{1}, N_{2}\right)}^{(n) 2}= & \left(N_{1}+1\right)\left(\ln \left(\sqrt{R_{1}^{(n)}}\right)\right)^{2} \\
& +\left(N_{2}+1\right)\left(\ln \left(\sqrt{R_{2}^{(n)}}\right)\right)^{2},
\end{aligned}
$$

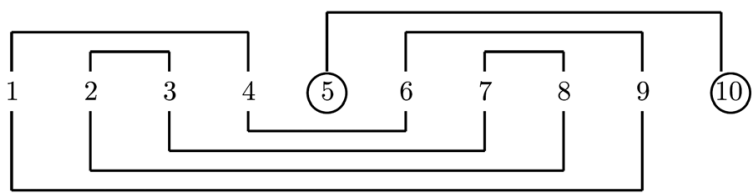

FIG. 4. $\quad N=10$ diagram.

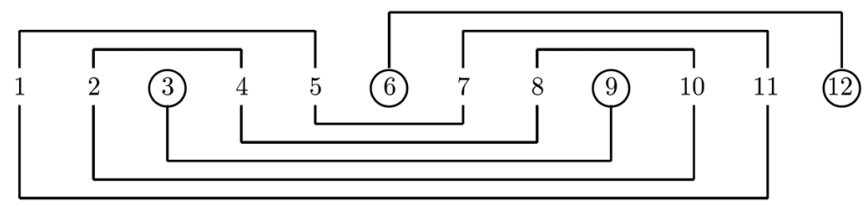

FIG. 5. $N=12$ diagram.

in which $R_{1}^{(n)}$ and $R_{2}^{(n)}$ are described in (3.34). In Sec. IV, we generalize it to the case of $N$ coupled oscillators that correspond to the lattice version of $\lambda \phi^{4}$ theory. We use a new kind of simple diagrams, Figs. 3-5, to represent the perturbative series and construct simple rules, Figs. 1 and 2, to calculate the complexity therein. We find that the diagrams are classified into three classes: odd $N$, odd $\frac{N}{2}$, and even $\frac{N}{2}$. We explicitly calculate the complexity in the cases of $N=2-5$ to any order of $\lambda$. Using these experiences, we then in Sec. V derive the general formulas of complexity in (5.5), (5.12), and (5.17). Then we present several diagrams to illuminate the properties of complexity and find that the interaction correction to complexity may be positive or negative depending on the magnitude of the reference-state frequency. We conclude in Sec. VI.

\section{INTERACTING SCALAR FIELD AND COUPLED OSCILLATORS}

The $d$-dimensional massive scalar Hamiltonian with a $\hat{\lambda} \phi^{4}$ interaction is

$$
H=\frac{1}{2} \int d^{d-1} x\left[\pi(x)^{2}+\vec{\nabla} \phi(x)^{2}+m^{2} \phi(x)^{2}+\frac{\hat{\lambda}}{12} \phi(x)^{4}\right] .
$$

Placing the theory on a square lattice with lattice spacing $\delta$, one has

$$
H=\frac{1}{2} \sum_{\vec{n}}\left\{\frac{p(\vec{n})^{2}}{\delta^{d-1}}+\delta^{d-1}\left[\frac{1}{\delta^{2}} \sum_{i}\left(\left(\phi(\vec{n})-\phi\left(\vec{n}-\hat{a}_{i}\right)\right)^{2}+m^{2} \phi(\vec{n})^{2}+\frac{\hat{\lambda}}{12} \phi(\vec{n})^{4}\right]\right\}\right.
$$

where $\hat{a}_{i}$ are unit vectors pointing toward the spatial directions of the lattice. By redefining

$$
X(\vec{n})=\delta^{d / 2} \phi(\vec{n}), \quad P(\vec{n})=\frac{p(\vec{n})}{\delta^{d / 2}}, \quad M=\frac{1}{\delta}, \quad \omega=m, \quad \Omega=\frac{1}{\delta}, \quad \lambda=\frac{\hat{\lambda}}{24 \delta^{4}},
$$

the Hamiltonian becomes 


$$
H=\sum_{\vec{n}}\left\{\frac{P(\vec{n})^{2}}{2 M}+\frac{1}{2} M\left[\omega^{2} X(\vec{n})^{2}+\Omega^{2} \sum_{i}\left(X(\vec{n})-X\left(\vec{n}-\hat{a}_{i}\right)\right)^{2}+2 \lambda X(\vec{n})^{4}\right]\right\} .
$$

When $\vec{n}$ is a one-dimensional vector, the Hamiltonian describes an infinite family of coupled one-dimensional oscillators. We will extensively study the one-dimensional oscillators in this paper, while the extension to higher dimensions is just to replace the site index $i$ to $\vec{i}$, as that described in [13].

\section{TWO COUPLED OSCILLATORS}

First we consider a simple case of two coupled oscillators $(M=1)$,

$$
H=\frac{1}{2}\left[\tilde{p}_{1}^{2}+\tilde{p}_{2}^{2}+\omega^{2}\left(\tilde{x}_{1}^{2}+\tilde{x}_{2}^{2}\right)+\Omega^{2}\left(\tilde{x}_{1}-\tilde{x}_{2}\right)^{2}+2 \lambda\left(\tilde{x}_{1}^{4}+\tilde{x}_{2}^{4}\right)\right] .
$$

Defining

$$
\tilde{x}_{1,2}=\frac{1}{\sqrt{2}}\left(x_{1} \pm x_{2}\right), \quad \tilde{p}_{1,2}=\frac{1}{\sqrt{2}}\left(p_{1} \pm p_{2}\right), \quad \omega_{1}^{2}=\omega^{2}, \quad \omega_{2}^{2}=\omega^{2}+2 \Omega^{2},
$$

the Hamiltonian is

$$
H=\frac{1}{2}\left(p_{1}^{2}+\omega_{1}^{2} x_{1}^{2}+p_{2}^{2}+\omega_{2}^{2} x_{2}^{2}\right)+\frac{\lambda}{4}\left(\left(x_{1}+x_{2}\right)^{4}+\left(x_{1}-x_{2}\right)^{4}\right)=K+V .
$$

In the second quantization, we define

$$
\begin{gathered}
a_{1}^{\dagger}=\sqrt{\frac{\omega_{1}}{2}} x_{1}+i \frac{1}{\sqrt{2 \omega_{1}}} p_{1}, \quad a_{2}^{\dagger}=\sqrt{\frac{\omega_{2}}{2}} x_{2}+i \frac{1}{\sqrt{2 \omega_{2}}} p_{2}, \quad\left[a_{1,2}, a_{1,2}^{\dagger}\right]=1, \\
x_{1,2}=\sqrt{\frac{1}{2 \omega_{1,2}}}\left(a_{1,2}^{\dagger}+a_{1,2}\right), \quad p_{1,2}=i \sqrt{\frac{\omega_{1,2}}{2}}\left(a_{1,2}^{\dagger}-a_{1,2}\right) .
\end{gathered}
$$

The state wave function is $\psi\left(x_{1}, x_{2}\right)=\left\langle x_{1}, x_{2}\left|a_{1}^{\dagger} a_{2}^{\dagger}\right| 0\right\rangle$.

\section{A. Kinetic term of two coupled oscillators}

The kinetic term has a diagonal form

$$
K^{(\operatorname{tar})}=\omega_{1} a_{1}^{\dagger} a_{1}+\omega_{2} a_{2}^{\dagger} a_{2}+\frac{1}{2}\left(\omega_{1}+\omega_{2}\right),
$$

where the constant terms are irrelevant to the following discussions. We choose the reference state with the associated kinetic term given by [13]

$$
K^{(\mathrm{ref})}=\omega_{f}\left(a_{1}^{(\mathrm{ref})}\right)^{\dagger} a_{1}^{(\mathrm{ref})}+\omega_{f}\left(a_{2}^{(\mathrm{ref})}\right)^{\dagger} a_{2}^{(\mathrm{ref})} .
$$

Now we see that, with the replacement

$$
a_{1}^{(\mathrm{ref})} \rightarrow \sqrt{\frac{\omega_{1}}{\omega_{f}}} a_{1}, \quad a_{2}^{(\mathrm{ref})} \rightarrow \sqrt{\frac{\omega_{2}}{\omega_{f}}} a_{2},
$$

one can obtain $K^{(\mathrm{tar})}$ from $K^{(\mathrm{ref})}$, i.e.,

$$
K^{(\mathrm{ref})} \rightarrow K^{(\mathrm{tar})}
$$

In the operator approach, the gate matrix defined in (1.1) is constructed by the transformation from target operator to reference operator in the above relation.

Consider first the ground state that is annihilated by $a_{1}, a_{2}$; i.e., $a_{1} a_{2}|0,0\rangle=0$ for the target state, and $a_{1}^{\text {(ref) }} a_{2}^{(\text {ref })}|0,0\rangle_{\text {ref }}=0$ for the reference state. The matrix $U(s)$ connecting the target operator with reference operator in $(1.1)$ is

$U(1)=\left(\begin{array}{cc}\sqrt{\frac{\omega_{1}}{\omega_{f}}} & 0 \\ 0 & \sqrt{\frac{\omega_{2}}{\omega_{f}}}\end{array}\right) \Rightarrow\left(\begin{array}{ll}a_{1} & a_{2}\end{array}\right)=U(1)\left(\begin{array}{c}a_{1}^{(\mathrm{ref})} \\ a_{2}^{(\mathrm{ref})}\end{array}\right)$

which leads to the replacement relation in (3.8), with initial condition $U(0)=\operatorname{diag}(1,1)$. Since the transformation matrix $U(1)$ is diagonal, we can choose $\mathcal{O}_{I}=1$ in (1.1) and have a simple relation 
$U(1)=\left(\begin{array}{cc}e^{\int_{0}^{1} d s Y_{1}(s)} & 0 \\ 0 & e^{\int_{0}^{1} d s Y_{2}(s)}\end{array}\right)=\left(\begin{array}{cc}\sqrt{\frac{\omega_{1}}{\omega_{f}}} & 0 \\ 0 & \sqrt{\frac{\omega_{2}}{\omega_{f}}}\end{array}\right)$.

The associated solutions of $Y_{(1,2)}(s)$ are

$$
Y_{1}(s)=\ln \left(\sqrt{\frac{\omega_{1}}{\omega_{f}}}\right), \quad Y_{2}(s)=\ln \left(\sqrt{\frac{\omega_{2}}{\omega_{f}}}\right),
$$

which satisfied the initial condition. The squared distance for the ground state, denoted as $D_{(0,0)}^{2}$, between target and reference states calculated from (1.2) becomes

$$
\begin{aligned}
D_{(0,0)}^{2} & =Y_{1}(1)^{2}+Y_{2}(1)^{2} \\
& =\left(\ln \left(\sqrt{\frac{\omega_{1}}{\omega_{f}}}\right)\right)^{2}+\left(\ln \left(\sqrt{\frac{\omega_{2}}{\omega_{f}}}\right)\right)^{2} .
\end{aligned}
$$

This matches with the result obtained earlier in [18].

Consider next the $\left\{N_{1}^{\text {th }} N_{2}^{\text {th }}\right\}$ excited state, which is defined by $a_{1}^{N_{1}+1} a_{2}^{N_{2}+1}\left|N_{1}, N_{2}\right\rangle=0 \quad$ or $\quad\left|N_{1}, N_{2}\right\rangle=$ $\frac{\left(a_{1}^{\dagger}\right)^{N_{1}+1}\left(a_{2}^{\dagger}\right)^{N_{2}+1}}{\sqrt{\left(N_{1}+1\right) !\left(N_{2}+1\right) !}}|0,0\rangle$. In this case, the gate matrices can be read from the transformations

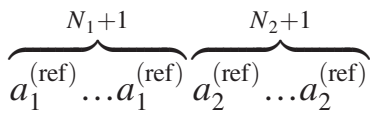

$$
\begin{aligned}
& \Rightarrow \overbrace{\sqrt{\frac{\omega_{1}}{\omega_{f}}} a_{1} \ldots \sqrt{\frac{\omega_{1}}{\omega_{f}}} a_{1}}^{N_{1}+1} \overbrace{\sqrt{\frac{\omega_{2}}{\omega_{f}}} a_{2} \ldots \sqrt{\frac{\omega_{2}}{\omega_{f}}} a_{2}}^{N_{2}+1}
\end{aligned}
$$

Then, the operator connecting the target operator with the reference operator in (1.1) becomes a $\left(N_{1}+1\right) \times\left(N_{2}+1\right)$ diagonal matrix $U(s)$ with elements

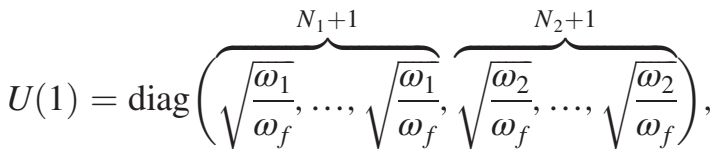

which becomes (3.10) in the case of the ground state $N_{1}=N_{2}=0$. Follow the discussions in before the matrix $U_{i}$ defined in (1.1) now becomes
$U_{i}(1)=\sqrt{\frac{\omega_{1}}{\omega_{f}}}, \quad$ with $\quad U_{i}(0)=1, \quad 1 \leq i \leq N_{1}+1$,

$$
\begin{array}{r}
U_{j}(1)=\sqrt{\frac{\omega_{2}}{\omega_{f}}}, \quad \text { with } \quad U_{j}(0)=1, \\
N_{1}+2 \leq j \leq N_{2}+N_{1}+2 .
\end{array}
$$

The associated functions of $Y_{i}(s)$ solved from (1.1) are

$$
\begin{gathered}
Y_{i}(s)=\ln \left(\sqrt{\frac{\omega_{1}}{\omega_{f}}}\right), \quad 1 \leq i \leq N_{1}+1, \\
Y_{j}(s)=\ln \left(\sqrt{\frac{\omega_{2}}{\omega_{f}}}\right), \quad N_{1}+2 \leq j \leq N_{2}+N_{1}+2 .
\end{gathered}
$$

The squared distance for the excited state, denoted as $D_{\left(N_{1}, N_{2}\right)}^{2}$, between target and reference states calculated from (1.2) is

$$
\begin{aligned}
& D_{\left(N_{1}, N_{2}\right)}^{2} \\
& =\sum_{i=1}^{N_{1}+1} Y_{i}(1)^{2}+\sum_{j=N_{1}+2}^{N_{1}+N_{2}+2} Y_{j}(1)^{2} \\
& =\left(N_{1}+1\right)\left(\ln \left(\sqrt{\frac{\omega_{1}}{\omega_{f}}}\right)\right)^{2}+\left(N_{2}+1\right)\left(\ln \left(\sqrt{\frac{\omega_{2}}{\omega_{f}}}\right)\right)^{2} .
\end{aligned}
$$

This matches with the result obtained earlier in [18].

Recalling that the state wave function is described by $\Psi_{n}(x)=\frac{1}{\sqrt{n !}}\left\langle x\left|\left(a^{\dagger}\right)^{n}\right| 0\right\rangle$, the gate matrix of excited-state wave function $\Psi_{n}(x)$ is thus related to the gate matrix of field operators $\left(a^{\dagger}\right)^{n}$.

\section{B. Interacting term of two coupled oscillators}

We next study the correction to the complexity due to the interaction term

$$
\begin{aligned}
V & =\frac{\lambda}{4}\left(\left(x_{1}+x_{2}\right)^{4}+\left(x_{1}-x_{2}\right)^{4}\right) \\
& =\frac{\lambda}{4 \cdot 2^{2}}\left[\left(\sqrt{\frac{1}{\omega_{1}}}\left(a_{1}^{\dagger}+a_{1}\right)+\sqrt{\frac{1}{\omega_{2}}}\left(a_{2}^{\dagger}+a_{2}\right)\right)^{4}\left(\sqrt{\frac{1}{\omega_{1}}}\left(a_{1}^{\dagger}+a_{1}\right)-\sqrt{\frac{1}{\omega_{2}}}\left(a_{2}^{\dagger}+a_{2}\right)\right)^{4}\right] \\
& =\frac{\lambda \cdot 2}{4 \cdot 2^{2}}\left[\left(\sqrt{\frac{1}{\omega_{1}}}\left(a_{1}^{\dagger}+a_{1}\right)\right)^{4}+\left(\sqrt{\frac{1}{\omega_{2}}}\left(a_{2}^{\dagger}+a_{2}\right)\right)^{4}+6\left(\sqrt{\frac{1}{\omega_{1}}}\left(a_{1}^{\dagger}+a_{1}\right)\right)^{2}\left(\sqrt{\frac{1}{\omega_{2}}}\left(a_{2}^{\dagger}+a_{2}\right)\right)^{2}\right] .
\end{aligned}
$$


We will consider $\left\langle N_{1}, N_{2}|V| N_{1}, N_{2}\right\rangle$ for the excited state $\left|N_{1}, N_{2}\right\rangle$ with fixed $N_{1}$ and $N_{2}$. In this way, only the terms that have the same power of $a_{i}$ and $a_{i}^{\dagger}$ are relevant. Therefore, we only need to consider

$$
\begin{aligned}
\left(a_{1}^{\dagger}+a_{1}\right)^{4} & =\left(\left(a_{1}^{\dagger}\right)^{2}+a_{1}^{\dagger} a_{1}+a_{1} a_{1}^{\dagger}+\left(a_{1}\right)^{2}\right)\left(\left(a_{1}^{\dagger}\right)^{2}+a_{1}^{\dagger} a_{1}+a_{1} a_{1}^{\dagger}+\left(a_{1}\right)^{2}\right) \\
& =6 a_{1}^{\dagger} a_{1} a_{1}^{\dagger} a_{1}+6 a_{1}^{\dagger} a_{1}+3+\text { irrelevant terms, } \\
\left(a_{1}^{\dagger}+a_{1}\right)^{2} & =\left(a_{1}^{\dagger}\right)^{2}+a_{1}^{\dagger} a_{1}+a_{1} a_{1}^{\dagger}+\left(a_{1}\right)^{2}=2 a_{1}^{\dagger} a_{1}+1+\text { irrelevant terms. }
\end{aligned}
$$

We obtain, after dropping irrelevant terms,

$$
H=\left(\omega_{1}+\frac{3 \lambda}{2}\left(\frac{1+N_{1}}{2 \omega_{1}^{2}}+\frac{1+N_{2}}{\omega_{1} \omega_{2}}\right)\right) a_{1}^{\dagger} a_{1}+\left(\omega_{2}+\frac{3 \lambda}{2}\left(\frac{1+N_{1}}{\omega_{2} \omega_{1}}+\frac{1+N_{2}}{2 \omega_{2}^{2}}\right)\right) a_{2}^{\dagger} a_{2} .
$$

The associated Hamiltonian of the reference state can be chosen as

$$
H^{(\mathrm{ref})}=\left(\omega_{f}+\frac{3 \lambda}{2} \frac{1+N_{1}}{2 \omega_{f}^{2}}\right)\left(a_{1}^{(\mathrm{ref})}\right)^{\dagger} a_{1}^{(\mathrm{ref})}+\left(\omega_{f}+\frac{3 \lambda}{2} \frac{1+N_{2}}{2 \omega_{f}^{2}}\right)\left(a_{2}^{(\mathrm{ref})}\right)^{\dagger} a_{2}^{(\mathrm{ref})},
$$

which satisfies a desirable property of the reference state, in that it does not contain any entanglement between operators $\left\{N_{1}, a_{1}^{(\text {ref })},\left(a_{1}^{(\text {ref })}\right)^{\dagger}\right\}$ and $\left\{N_{2}, a_{2}^{(\text {ref })},\left(a_{2}^{(\text {ref })}\right)^{\dagger}\right\}$. The property is like that in the coordinate approach, in which a desirable property of the reference state is that it should not contain any entanglement between the original coordinates $x_{1}$ and $x_{2}$ [17].

In the case of zero order of $\lambda$,

$$
K^{(\mathrm{ref})}=\omega_{f}\left(a_{1}^{(\mathrm{ref})}\right)^{\dagger} a_{1}^{(\mathrm{ref})}+\omega_{f}\left(a_{2}^{(\mathrm{ref})}\right)^{\dagger} a_{2}^{(\mathrm{ref})}, \quad K^{(\mathrm{tar})}=\omega_{1} a_{1}^{\dagger} a_{1}+\omega_{1} a_{2}^{\dagger} a_{2},
$$

which implies transformations

$$
\begin{gathered}
a_{1}^{(\mathrm{ref})} \rightarrow \sqrt{\frac{\omega_{1}}{\omega_{f}}} a_{1}^{(\mathrm{ref})}, \quad a_{2}^{(\mathrm{ref})} \rightarrow \sqrt{\frac{\omega_{2}}{\omega_{f}}} a_{2}^{(\mathrm{ref})} \Rightarrow K^{(\mathrm{ref})} \rightarrow K^{(\mathrm{tar})} \\
\text { or } \quad N_{(1,2)}^{(\mathrm{ref})} \rightarrow R_{(1,2)}^{(0)} N_{(1,2)}^{(\mathrm{ref})} \Rightarrow K^{(\mathrm{ref})} \rightarrow K^{(\mathrm{tar})},
\end{gathered}
$$

where

$$
R_{i}^{(0)}=\frac{\omega_{i}}{\omega_{f}}
$$

The quantum gate is described by two $1 \times 1$ matrices, $\exp \left(\sqrt{R_{1}^{(0)}}\right)$ and $\exp \left(\sqrt{R_{2}^{(0)}}\right)$. This is the case of a purely kinetic term, i.e., a free theory.

Now consider a perturbation to the complexity for the two coupled oscillators. At the first order of $\lambda$, we have transformations

$$
\begin{cases}\left(\omega_{1}+\frac{3 \lambda}{2}\left(\frac{1+N_{1}}{2 \omega_{1}^{2}}+\frac{1+N_{2}}{\omega_{1} \omega_{2}}\right)\right) a_{1}^{\dagger} a_{1} \rightarrow\left(\omega_{f}+\frac{3 \lambda}{2} \frac{1+N_{1}}{2 \omega_{f}^{2}}\right)\left(a_{1}^{(\mathrm{ref})}\right)^{\dagger} a_{1}^{(\mathrm{ref})} \\ \left(\omega_{2}+\frac{3 \lambda}{2}\left(\frac{1+N_{1}}{\omega_{2} \omega_{1}}+\frac{1+N_{2}}{2 \omega_{2}^{2}}\right)\right) a_{2}^{\dagger} a_{2} \rightarrow\left(\omega_{f}+\frac{3 \lambda}{2} \frac{1+N_{2}}{2 \omega_{f}^{2}}\right)\left(a_{2}^{(\mathrm{ref})}\right)^{\dagger} a_{2}^{(\mathrm{ref})} .\end{cases}
$$

The factors $N_{(1,2)}$ are within the coupling term, i.e., $\frac{3 \lambda}{2}$, and we only need to consider their zero-order transform. Recalling (3.28), we have to multiple them by $R_{(1,2)}^{(0)}$ factors. Therefore, the first-order transformations are 


$$
R_{1}^{(1)}=\frac{\omega_{1}+\frac{3 \lambda}{2}\left(\frac{1+N_{1} R_{1}^{(0)}}{2 \omega_{1}^{2}}+\frac{1+N_{2} R_{2}^{(0)}}{\omega_{1} \omega_{2}}\right)}{\omega_{f}+\frac{3 \lambda}{2} \frac{1+N_{1}}{2 \omega_{f}^{2}}}, \quad R_{2}^{(1)}=\frac{\omega_{2}+\frac{3 \lambda}{2}\left(\frac{1+N_{1} R_{1}^{(0)}}{\omega_{2} \omega_{1}}+\frac{1+N_{2} R_{2}^{(0)}}{2 \omega_{2}^{2}}\right)}{\omega_{f}+\frac{3 \lambda}{2} \frac{1+N_{2}}{2 \omega_{f}^{2}}} .
$$

Now, the functions $\left(R_{1}^{(1)}, R_{2}^{(1)}\right)$ play the roles of $\left(\frac{\omega_{1}}{\omega_{f}}, \frac{\omega_{2}}{\omega_{f}}\right)$ in free theory, i.e., (3.29), and the square distance formula (3.13) becomes

$$
D_{(0,0)}^{(1) 2}=\left(\ln \left(\sqrt{R_{1}^{(1)}}\right)\right)^{2}+\left(\ln \left(\sqrt{R_{2}^{(1)}}\right)\right)^{2}
$$

For excited states, along the same analysis in free theory, the first-order square distance is

$$
D_{\left(N_{1}, N_{2}\right)}^{2}=\left(N_{1}+1\right)\left(\ln \left(\sqrt{R_{1}^{(1)}}\right)\right)^{2}+\left(N_{2}+1\right)\left(\ln \left(\sqrt{R_{2}^{(1)}}\right)\right)^{2}
$$

Extending to higher-order interactions is straightforward. The recursion relations are

$$
R_{1}^{(n)}=\frac{\omega_{1}+\frac{3 \lambda}{2}\left(\frac{1+N_{1} R_{1}^{(n-1)}}{2 \omega_{1}^{2}}+\frac{1+N_{2} R_{2}^{(n-1)}}{\omega_{1} \omega_{2}}\right)}{\omega_{f}+\frac{3 \lambda}{2} \frac{1+N_{1}}{2 \omega_{f}^{2}}}, \quad R_{2}^{(n)}=\frac{\omega_{2}+\frac{3 \lambda}{2}\left(\frac{1+N_{1} R_{1}^{(n-1)}}{\omega_{2} \omega_{1}}+\frac{1+N_{2} R_{2}^{(n-1)}}{2 \omega_{2}^{2}}\right)}{\omega_{f}+\frac{3 \lambda}{2} \frac{1+N_{2}}{2 \omega_{f}^{2}}}
$$

with initial values $R_{(1,2)}^{(0)}$ defined in (3.29). For excited states, the $n$-order square distance is

$$
D_{\left(N_{1}, N_{2}\right)}^{(n) 2}=\left(N_{1}+1\right)\left(\ln \left(\sqrt{R_{1}^{(n)}}\right)\right)^{2}+\left(N_{2}+1\right)\left(\ln \left(\sqrt{R_{2}^{(n)}}\right)\right)^{2},
$$

which is the $n$-order complexity of two coupled oscillators.

Note that original relations (3.34) can be expanded as

$$
\begin{aligned}
& R_{1}^{(n)} \approx \frac{\omega_{1}}{\omega_{f}}+\frac{3 \lambda}{2 \omega_{f}}\left(\frac{1+N_{1} R_{1}^{(n-1)}}{2 \omega_{1}^{2}}+\frac{1+N_{2} R_{2}^{(n-1)}}{\omega_{1} \omega_{2}}-\frac{1+N_{1}}{2 \omega_{f}^{2}}\right), \\
& R_{2}^{(n)} \approx \frac{\omega_{2}}{\omega_{f}}+\frac{3 \lambda}{2 \omega_{f}}\left(\frac{1+N_{1} R_{1}^{(n-1)}}{\omega_{2} \omega_{1}}+\frac{1+N_{2} R_{2}^{(n-1)}}{2 \omega_{2}^{2}}-\frac{1+N_{2}}{2 \omega_{f}^{2}}\right) .
\end{aligned}
$$

In this way, the perturbative series of $R_{i}^{(n)}$ in coupling constant $\lambda$ is explicitly showing up. However, to save the space, in following sections we will not expand the original relations, like as (3.34), to the relations, like as (3.36) or (3.37).

\section{N COUPLED OSCILLATORS}

\section{A. Kinetic term of $N$ coupled oscillators}

For $N$ coupled oscillators,

$H=\frac{1}{2} \sum_{k=1}^{N}\left[\tilde{p}_{k}^{2}+\omega^{2} \tilde{x}_{k}^{2}+\Omega^{2}\left(\tilde{x}_{k}-\tilde{x}_{k+1}\right)^{2}+2 \lambda \tilde{x}_{k}^{4}\right]$.

We impose a periodic boundary condition $\tilde{x}_{k+N+1}=\tilde{x}_{k}$. The normal coordinates are chosen to be

$$
\begin{aligned}
& x_{k}=\frac{1}{\sqrt{N}} \sum_{j=1}^{N} \exp \left(\frac{2 \pi i k}{N} j\right) \tilde{x}_{j}, \\
& p_{k}=\frac{1}{\sqrt{N}} \sum_{j=1}^{N} \exp \left(\frac{-2 \pi i k}{N} j\right) \tilde{p}_{j} .
\end{aligned}
$$

Note that the relative sign between the Fourier series of $x_{k}$ and $p_{k}$ is important to have standard commutation relation $\left[x_{k_{1}}, p_{k_{2}}\right]=\delta_{k_{1}, k_{2}}$ [13]. The Hamiltonian now becomes

$$
\begin{aligned}
H & =\frac{1}{2} \sum_{k=1}^{N}\left(p_{k}^{\dagger} p_{k}+\omega_{k}^{2} x_{k}^{\dagger} x_{k}\right)+V, \\
\omega_{k}^{2} & =\omega^{2}+4 \Omega^{2} \sin ^{2} \frac{\pi k}{N} .
\end{aligned}
$$

Defining 


$$
\begin{aligned}
x_{k} & =\frac{1}{\sqrt{2 \omega_{k}}}\left(a_{k}+a_{-k}^{\dagger}\right), \quad p_{k}=i \sqrt{\frac{\omega_{k}}{2}}\left(a_{k}^{\dagger}-a_{-k}\right), \\
{\left[a_{k}, a_{k}^{\dagger}\right] } & =1,
\end{aligned}
$$

the kinetic term can be written as

$$
\sum_{k} K_{k}=\frac{1}{2} \sum_{k}\left(p_{k}^{\dagger} p_{k}+\omega_{k}^{2} x_{k}^{\dagger} x_{k}\right)=\sum_{k} \omega_{k} a_{k}^{\dagger} a_{k},
$$

up to an irrelevant constant.

The states in $N$ oscillators can be defined by the creation operators $a_{1}^{\dagger} a_{2}^{\dagger} \ldots a_{k}^{\dagger} \ldots$, such that $\psi\left(x_{1}, x_{2}, \ldots\right)=$ $\left\langle x_{1}, x_{2}, \ldots x_{k} \ldots\left|a_{1}^{\dagger} a_{2}^{\dagger} \ldots a_{k}^{\dagger} \ldots\right| 0\right\rangle$. As before, to find the complexity of such state we choose a reference state with the associated kinetic term given by

$$
K^{(\mathrm{ref})}=\sum_{k} \omega_{f}\left(a_{k}^{(\mathrm{ref})}\right)^{\dagger} a_{k}^{(\mathrm{ref})} .
$$

The square distance for the $n_{k}$ th excited state is

$$
\begin{aligned}
D_{\left\{N_{1}, N_{2}, \ldots N_{k}, \ldots N_{N}\right\}}^{2}= & \left(N_{1}+1\right)\left(\ln \left(\sqrt{\frac{\omega_{1}}{\omega_{f}}}\right)\right)^{2} \\
& +\left(N_{2}+1\right)\left(\ln \left(\sqrt{\frac{\omega_{2}}{\omega_{f}}}\right)\right)^{2}+\ldots \\
= & \sum_{k=1}^{N}\left(N_{k}+1\right)\left(\ln \left(\sqrt{\frac{\omega_{k}}{\omega_{f}}}\right)\right)^{2},
\end{aligned}
$$

where $\omega_{k}$ is defined in (4.3).

\section{B. Interacting term of $N$ coupled oscillators: Perturbative algorithm}

We adopt the following steps to systematically study a perturbation theory of the complexity:

(I) We express potential $V$ in terms of $a, a^{\dagger}$

$$
\begin{aligned}
V= & \lambda \sum_{k=1}^{N} \tilde{x}_{k}^{4}=\lambda \sum_{k=1}^{N}\left(\frac{1}{\sqrt{N}} \sum_{j=1}^{N} \exp \left(\frac{-2 \pi i k}{N} j\right) x_{j}\right)^{4} \\
= & \frac{\lambda}{4 N^{2}} \sum_{k=1}^{N}\left(\sum_{j=1}^{N} \exp \left(\frac{-2 \pi i k}{N} j\right)\right. \\
& \left.\times\left(\frac{1}{\sqrt{\omega_{j}}}\left(a_{j}+a_{j}^{\dagger}\right)\right)\right)^{4}
\end{aligned}
$$

(II) Define

$$
A(j)=\frac{1}{\sqrt{\omega_{j}}}\left(a_{j}+a_{j}^{\dagger}\right) .
$$

Then, as calculated in (3.22) and (3.23),
$A(j)^{4}=\frac{6}{\omega_{j}^{2}}\left(a_{j}^{\dagger} a_{j} a_{j}^{\dagger} a_{j}+a_{j}^{\dagger} a_{j}\right)+$ irrelevant terms,

$A(j)^{2}=\frac{1}{\omega_{j}}\left(2 a_{j}^{\dagger} a_{j}+1\right)+$ irrelevant terms,

which lead to two relations that will be extensively used in later calculations ${ }^{2}$

$$
A(j)^{4}=6 \times\left[\frac{N_{j}+1}{\omega_{j}^{2}} a_{j}^{\dagger} a_{j}\right],
$$

$$
\begin{aligned}
& 6 A(i)^{2} A(j)^{2} \\
& =6 \times \frac{1}{\omega_{i} \omega_{j}}\left(4 a_{i}^{\dagger} a_{i} a_{j}^{\dagger} a_{j}+2 a_{i}^{\dagger} a_{i}+2 a_{j}^{\dagger} a_{j}\right) \\
& =6 \times\left[\frac{2 N_{i}+2}{\omega_{i} \omega_{j}} a_{j}^{\dagger} a_{j}+\frac{2 N_{j}+2}{\omega_{i} \omega_{j}} a_{i}^{\dagger} a_{i}\right], \quad i \neq j .
\end{aligned}
$$

The term $a_{j}^{\dagger} a_{j} a_{j}^{\dagger} a_{j}$ in (4.10) is written as $N_{j} a_{j}^{\dagger} a_{j}$ in (4.12), as we did in Sec. III. B. Section III. B also tells us that we will let $N_{j} \rightarrow R_{j}^{(n-1)} N_{j}$ in calculating the complexity at the nth order of $\lambda$.

(III) Adopting the series expansion (4.8), we can develop diagrammatic rules based on two basic elements, "circle" and "pair," which appear in (4.12) and (4.13). We plot them in Figs. 1 and 2.

(IV) The diagrams for the potential $V$ then can be classified into three classes: odd $N$, odd $\frac{N}{2}$, and even $\frac{N}{2}$. We discuss corresponding rules in the following.

(i) Odd $N$ : We write numbers $1,2, \ldots, N$ on a horizon and assign a circle on $N$. Then we assign a pair on $(1, N-1), \ldots,(i, N-i), \ldots,\left(\frac{N-1}{2}, \frac{N+1}{2}\right)$.

An $N=9$ example is plotted in Fig. 3.

(ii) Odd $\frac{N}{2}$ : We write numbers $1,2, \ldots, N$ on a horizon and assign a circle on $\frac{N}{2}$ and on $N$. We also assign a pair on $\left(\frac{N}{2}, N\right)$, on $(1, N-1), \ldots,(i, N-i), \ldots$, $\left(\frac{N}{2}-1, \frac{N}{2}+1\right)$, and on $\left(1, \frac{N}{2}-1\right), \ldots,\left(i, \frac{N}{2}-i\right), \ldots$, $\left(\frac{N}{4}-\frac{1}{2}, \frac{N}{4}+\frac{1}{2}\right)$. Finally, we assign a pair on $\left(\frac{N}{2}+1, N-1\right), \ldots,\left(i, \frac{3 N}{2}-i\right), \ldots,\left(\frac{3 N}{4}-\frac{1}{2}, \frac{3 N}{4}+\frac{1}{2}\right)$.

An $N=10$ example is plotted in Fig. 4.

(iii) Even $\frac{N}{2}$ : We write numbers $1,2, \ldots, N$ on a horizon and assign a circle on $N, \frac{N}{4}, \frac{N}{2}$, and $\frac{3 N}{4}$. We also assign a pair on $\left(\frac{N}{2}, N\right)$ and $\left(\frac{N}{4}, \frac{3 N}{4}\right)$, on $(1, N-1), \ldots$, $(i, N-i), \ldots,\left(\frac{N}{2}-1, \frac{N}{2}+1\right)$, and on $\left(1, \frac{N}{2}-1\right), \ldots$, $\left(i, \frac{N}{2}-i\right), \ldots,\left(\frac{N}{4}-1, \frac{N}{4}+1\right)$. Finally, we assign a

\footnotetext{
${ }^{2}$ The reason for using $6 A(i)^{2} A(j)^{2}$ instead of $A(i)^{2} A(j)^{2}$ is because, in the series expansion of the potential (4.8), it always appears the combination factor $6 A(i)^{2} A(j)^{2}$, as can be seen in several examples in next subsection.
} 
pair on $\left(\frac{N}{2}+1, N-1\right), \ldots,\left(i, \frac{3 N}{2}-i\right), \ldots,\left(\frac{3 N}{4}-\right.$ $\left.1, \frac{3 N}{4}+1\right)$

An $N=12$ example is plotted in Fig. 5.

From Figs. 3-5, we can see a pairing property: Assign the circle element $A(j)^{4}$ pairing with " $\mathrm{j}$ " once and assign the pair element $A(i)^{2} A(j)^{2}$ pairing with each $i$ and $j$ once, then the odd $N$ diagrams have pairings in each $j$ once, while the even $N$ diagrams have pairings for each $j$ twice.

\section{Interacting term of $N$ coupled oscillators: Some calculations}

We now take several values of $N$ as examples to plot the diagrams and use (4.12) and (4.13) to calculate the associated complexity. General formulas will be presented in the next section.

(i) $N=2$ :

As shown in Fig. 6, the series expansion (4.8) is

$$
\begin{aligned}
V_{N=2} & =\frac{\lambda}{4 \cdot 2}\left[A(1)^{4}+A(2)^{4}+6 A(1)^{2} A(2)^{2}\right] \\
& =\frac{6 \lambda}{4 \cdot 2}\left[\frac{1}{\omega_{1}^{2}}\left(N_{1}+1\right) a_{1}^{\dagger} a_{1}+\frac{1}{\omega_{2}^{2}}\left(N_{2}+1\right) a_{2}^{\dagger} a_{2}+\frac{2}{\omega_{1} \omega_{2}}\left(\left(N_{2}+1\right) a_{1}^{\dagger} a_{1}+\left(N_{1}+1\right) a_{2}^{\dagger} a_{2}\right)\right] \\
& =\frac{6 \lambda}{4 \cdot 2}\left[\left(\frac{1+N_{1}}{\omega_{1}^{2}}+\frac{2+2 N_{2}}{\omega_{1} \omega_{2}}\right) a_{1}^{\dagger} a_{1}+\left(\frac{\left(1+N_{2}\right.}{\omega_{2}^{2}}+\frac{2+2 N_{1}}{\omega_{1} \omega_{2}}\right) a_{2}^{\dagger} a_{2}\right] .
\end{aligned}
$$

We have used (4.12) and (4.13). The above result matches with (3.24).

The associated complexity can be evaluated to any order in $\lambda$,

$$
R_{1}^{(n)}=\frac{\omega_{1}+\frac{6 \lambda}{4 \cdot 2}\left(\frac{1+N_{1} R_{1}^{(n-1)}}{\omega_{1}^{2}}+\frac{2+2 N_{2} R_{2}^{(n-1)}}{\omega_{1} \omega_{2}}\right)}{\omega_{f}+\frac{6 \lambda}{4 \cdot 2} \frac{1+N_{1}}{\omega_{f}^{2}}}, \quad R_{2}^{(n)}=\frac{\omega_{2}+\frac{6 \lambda}{4 \cdot 2}\left(\frac{1+N_{2} R_{2}^{(n-1)}}{\omega_{2}^{2}}+\frac{2+2 N_{1} R_{1}^{(n-1)}}{\omega_{1} \omega_{2}}\right)}{\omega_{f}+\frac{6 \lambda}{4 \cdot 2} \frac{2+2 N_{1}}{\omega_{f}^{2}}},
$$

with initial values $R_{(1,2)}^{(0)}$ defined in (3.29). For excited states, the $n$-order squared distance is $D_{\left(N_{1}, N_{2}\right)}^{(n) 2}=$ $\sum_{i=1}^{2}\left(N_{i}+1\right)\left(\ln \left(\sqrt{R_{i}^{(n)}}\right)\right)^{2}$, which is the $n$-order complexity of two coupled oscillators. While the above results exactly match (3.34), we have expressed them in the new form that helps us to identify rules for computing a general $N$ result.

(ii) $N=3$ :

As shown in Fig. 7, the series expansion (4.8) is

$$
\begin{aligned}
V_{N=3} & =\frac{\lambda}{4 \cdot 3}\left[A(3)^{4}+6 A(1)^{2} A(2)^{2}\right] \\
& =\frac{6 \lambda}{4 \cdot 3}\left[\frac{1+N_{3}}{\omega_{3}^{2}} a_{3}^{\dagger} a_{3}+\frac{2+2 N_{2}}{\omega_{1} \omega_{2}} a_{1}^{\dagger} a_{1}+\frac{2+2 N_{1}}{\omega_{1} \omega_{2}} a_{2}^{\dagger} a_{2}\right] .
\end{aligned}
$$

We have recurrent relations

$$
\begin{aligned}
& R_{1}^{(n)}=\frac{\omega_{1}+\frac{6 \lambda}{4 \cdot 3}\left(\frac{2+2 N_{2} R_{2}^{(n-1)}}{\omega_{1} \omega_{2}}\right)}{\omega_{f}+\frac{6 \lambda}{4 \cdot 3}\left(\frac{2+2 N_{2}}{\omega_{f}^{2}}\right)}, \quad R_{2}^{(n)}=\frac{\omega_{2}+\frac{6 \lambda}{4 \cdot 3}\left(\frac{2+2 N_{1} R_{1}^{(n-1)}}{\omega_{1} \omega_{2}}\right)}{\omega_{f}+\frac{6 \lambda}{4 \cdot 3}\left(\frac{2+2 N_{1}}{\omega_{f}^{2}}\right)}, \\
& R_{3}^{(n)}=\frac{\omega_{3}+\frac{6 \lambda}{4 \cdot 3}\left(\frac{1+N_{3} R_{3}^{(n-1)}}{\omega_{3}^{2}}\right)}{\omega_{f}+\frac{6 \lambda}{4 \cdot 3}\left(\frac{1+N_{3}}{\omega_{f}^{2}}\right)} .
\end{aligned}
$$

For excited states, $D_{\left(N_{1}, N_{2}, N_{3}\right)}^{(n) 2}=\sum_{i=1}^{3}\left(N_{i}+1\right)\left(\ln \left(\sqrt{R_{i}^{(n)}}\right)\right)^{2}$, which is the $n$-order complexity of three coupled oscillators.

(iii) $N=4$ :

As shown in Fig. 8, the series expansion (4.8) is 


$$
\begin{aligned}
V_{N=4}= & \frac{\lambda}{4 \cdot 4}\left[A(1)^{4}+A(2)^{4}+A(3)^{4}+A(4)^{4}+6 A(1)^{2} A(3)^{2}+6 A(2)^{2} A(4)^{2}\right] \\
= & \frac{6 \lambda}{4 \cdot 4}\left[\left(\frac{1+N_{1}}{\omega_{1}^{2}}+\frac{2+2 N_{3}}{\omega_{1} \omega_{3}}\right) a_{1}^{\dagger} a_{1}+\left(\frac{1+N_{2}}{\omega_{2}^{2}}+\frac{2+2 N_{4}}{\omega_{2} \omega_{4}}\right) a_{2}^{\dagger} a_{2}\right. \\
& \left.+\left(\frac{1+N_{3}}{\omega_{3}^{2}}+\frac{2+2 N_{1}}{\omega_{1} \omega_{3}}\right) a_{3}^{\dagger} a_{3}+\left(\frac{1+N_{4}}{\omega_{4}^{2}}+\frac{2+2 N_{2}}{\omega_{2} \omega_{4}}\right) a_{4}^{\dagger} a_{4}\right] .
\end{aligned}
$$

We have recurrent relations

$$
\begin{array}{lll}
R_{1}^{(n)}=\frac{\omega_{1}+\frac{6 \lambda}{4 \cdot 4}\left(\frac{1+N_{1} R_{1}^{(n-1)}}{\omega_{1}^{2}}+\frac{2+2 N_{3} R_{3}^{(n-1)}}{\omega_{1} \omega_{3}}\right)}{\omega_{f}+\frac{6 \lambda}{4 \cdot 4} \frac{1+N_{1}}{\omega_{f}^{2}}}, & R_{2}^{(n)}=\frac{\omega_{2}+\frac{6 \lambda}{4 \cdot 4}\left(\frac{1+N_{2} R_{2}^{(n-1)}}{\omega_{2}^{2}}+\frac{2+2 N_{4} R_{4}^{(n-1)}}{\omega_{2} \omega_{4}}\right)}{\omega_{f}+\frac{6 \lambda}{4 \cdot 4} \frac{2+2 N_{4}}{\omega_{f}^{2}}}, \\
R_{3}^{(n)}=\frac{\omega_{3}+\frac{6 \lambda}{4 \cdot 4}\left(\frac{1+N_{3} R_{3}^{(n-1)}}{\omega_{3}^{2}}+\frac{2+2 N_{1} R_{1}^{(n-1)}}{\omega_{1} \omega_{3}}\right)}{\omega_{f}+\frac{6 \lambda}{4 \cdot 4} \frac{1+N_{3}}{\omega_{f}^{2}}}, & R_{4}^{(n)}=\frac{\omega_{4}+\frac{6 \lambda}{4 \cdot 4}\left(\frac{1+N_{4} R_{4}^{(n-1)}}{\omega_{4}^{2}}+\frac{2+2 N_{2} R_{2}^{(n-1)}}{\omega_{2} \omega_{4}}\right)}{\omega_{f}+\frac{6 \lambda}{4 \cdot 4} \frac{1+N_{4}}{\omega_{f}^{2}}} .
\end{array}
$$

For excited states, $D_{\left(N_{1}, N_{2}, N_{3}, N_{4}\right)}^{(n) 2}=\sum_{i=1}^{4}\left(N_{i}+1\right)\left(\ln \left(\sqrt{R_{i}^{(n)}}\right)\right)^{2}$, which is the $n$-order complexity of four coupled oscillators.

(iv) $N=5$ :

As shown in Fig. 9, the series expansion (4.8) is

$$
\begin{gathered}
V_{N=5}=\frac{\lambda}{4 \cdot 5}\left[6 A(1)^{2} A(4)^{2}+6 A(2)^{2} A(3)^{2}+A(5)^{4}\right] \\
=\frac{6 \lambda}{4 \cdot 3}\left[\frac{1+N_{5}}{\omega_{5}^{2}} a_{5}^{\dagger} a_{5}+\frac{2+2 N_{4}}{\omega_{1} \omega_{4}} a_{1}^{\dagger} a_{1}+\frac{2+2 N_{3}}{\omega_{3} \omega_{2}} a_{2}^{\dagger} a_{2}+\frac{2+2 N_{2}}{\omega_{3} \omega_{2}} a_{3}^{\dagger} a_{3}+\frac{2+2 N_{1}}{\omega_{1} \omega_{4}} a_{4}^{\dagger} a_{4}\right] .
\end{gathered}
$$

We have recurrent relations

$$
\begin{aligned}
& R_{1}^{(n)}=\frac{\omega_{1}+\frac{6 \lambda}{4 \cdot 3}\left(\frac{2+2 N_{4} R_{4}^{(n-1)}}{\omega_{1} \omega_{4}}\right)}{\omega_{f}+\frac{6 \lambda}{4 \cdot 3}\left(\frac{2+2 N_{4}}{\omega_{f}^{2}}\right)}, \quad R_{2}^{(n)}=\frac{\omega_{2}+\frac{6 \lambda}{4 \cdot 3}\left(\frac{2+2 N_{3} R_{3}^{(n-1)}}{\omega_{3} \omega_{2}}\right)}{\omega_{f}+\frac{6 \lambda}{4 \cdot 3}\left(\frac{2+2 N_{3}}{\omega_{f}^{2}}\right)}, \\
& R_{3}^{(n)}=\frac{\omega_{3}+\frac{6 \lambda}{4 \cdot 3}\left(\frac{2+2 N_{2} R_{2}^{(n-1)}}{\omega_{3} \omega_{2}}\right)}{\omega_{f}+\frac{6 \lambda}{4 \cdot 3}\left(\frac{2+2 N_{2}}{\omega_{f}^{2}}\right)}, \quad R_{4}^{(n)}=\frac{\omega_{4}+\frac{6 \lambda}{4 \cdot 3}\left(\frac{2+2 N_{1} R_{1}^{(n-1)}}{\omega_{1} \omega_{4}}\right)}{\omega_{f}+\frac{6 \lambda}{4 \cdot 3}\left(\frac{2+2 N_{1}}{\omega_{f}^{2}}\right)}, \\
& R_{5}^{(n)}=\frac{\omega_{5}+\frac{6 \lambda}{4 \cdot 3}\left(\frac{1+N_{5} R_{5}^{(n-1)}}{\omega_{5}^{2}}\right)}{\omega_{f}+\frac{6 \lambda}{4 \cdot 3}\left(\frac{1+N_{5}}{\omega_{f}^{2}}\right)} .
\end{aligned}
$$

For excited states, $D_{\left(N_{1}, N_{2}, N_{3}\right)}^{(n) 2}=\sum_{i=1}^{5}\left(N_{i}+1\right)\left(\ln \left(\sqrt{R_{i}^{(n)}}\right)\right)^{2}$, which is the $n$-order complexity of five coupled oscillators.

(v) $N=6$ :

As shown in Fig. 10, the series expansion (4.8) is

$$
\begin{aligned}
V_{N=6}= & \frac{\lambda}{4 \cdot 6}\left[A(3)^{4}+A(6)^{4}+6 A(1)^{2} A(5)^{2}+6 A(2)^{2} A(4)^{2}+6 A(1)^{2} A(2)^{2}\right. \\
& \left.+6 A(3)^{2} A(6)^{2}+6 A(4)^{2} A(5)^{2}\right] .
\end{aligned}
$$




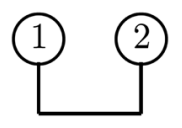

FIG. 6. $\quad N=2$ diagram.

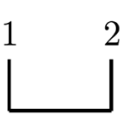

FIG. 7. $N=3$ diagram.

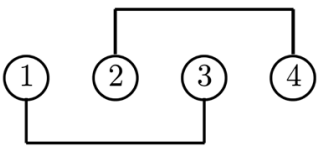

FIG. 8. $\quad N=4$ diagram.

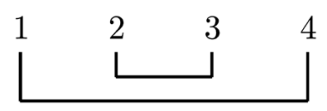

(5)

FIG. 9. $N=5$ diagram.

(vi) $N=7$ :

As shown in Fig. 11, the series expansion (4.8) is

$$
\begin{aligned}
V_{N=7}= & \frac{\lambda}{4 \cdot 7}\left[6 A(1)^{2} A(6)^{2}+6 A(2)^{2} A(5)^{2}\right. \\
& \left.+6 A(3)^{2} A(4)^{2}+A(7)^{4}\right] .
\end{aligned}
$$

(vii) $N=8$ :

As shown in Fig. 12, the series expansion (4.8) is

$$
\begin{aligned}
V_{N=8}= & \frac{\lambda}{4 \cdot 8}\left[A(2)^{4}+A(4)^{4}+A(6)^{4}+A(8)^{4}\right. \\
& +6 A(1)^{2} A(7)^{2}+6 A(2)^{2} A(6)^{2} \\
& +6 A(3)^{2} A(5)^{2}+6 A(1)^{2} A(3)^{2} \\
& \left.+6 A(4)^{2} A(8)^{2}+6 A(5)^{2} A(7)^{2}\right] .
\end{aligned}
$$

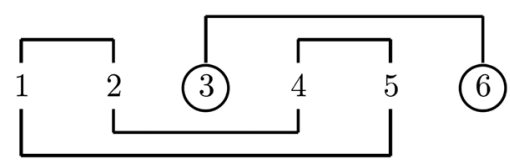

FIG. 10. $N=6$ diagram.

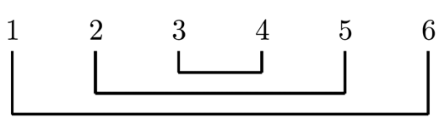

(7)

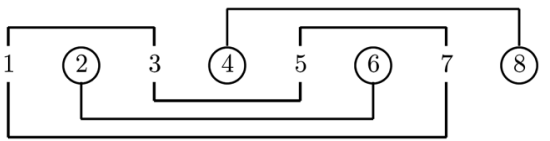

FIG. 12. $\quad N=8$ diagram.

With these experiences, we will in the next section derive general formulas of the complexity for any $N$ to any order in $\lambda$.

\section{COMPLEXITY OF $N$ COUPLED OSCILLATORS}

\section{A. Complexity of $N$ coupled oscillators: General formulas}

From the above analysis and relations (4.12) and (4.13), we find

$$
\begin{aligned}
V_{N} & =\frac{\lambda}{4 \cdot N}\left[\sum_{\text {circle } j} A(j)^{4}+6 \sum_{\text {pair }(i, j)} A(i)^{2} A(j)^{2}\right] \\
& =\frac{6 \lambda}{4 \cdot N}\left[\sum_{\text {circle } i} \frac{\left(1+N_{j}\right)}{\omega_{j}^{2}} a_{j}^{\dagger} a_{j}\right. \\
& \left.+\sum_{\text {pair }(i, j)} \frac{2+2 N_{i}}{\omega_{i} \omega_{j}} a_{j}^{\dagger} a_{j}+\frac{2+2 N_{j}}{\omega_{i} \omega_{j}} a_{i}^{\dagger} a_{i}\right],
\end{aligned}
$$

where circle and pair can be read from diagrams; see Figs. 3-5

(i) Odd $N$ : We recall, from $\operatorname{Sec} 4.2$, the odd $N$ case is simplest as it only has one circle located at $N$, and each pair is independent of each other (Fig. 3). Equation (5.1) becomes

$$
V_{\text {odd } N}=\frac{6 \lambda}{4 \cdot N}\left[\frac{1+N_{N}}{\omega_{N}^{2}} a_{N}^{\dagger} a_{N}+\sum_{i=1}^{N-1} \frac{2+2 N_{N-i}}{\omega_{i} \omega_{N-i}} a_{i}^{\dagger} a_{i}\right] .
$$

By adding the kinematic term (4.5) and defining the recursion relations

$$
R_{N, \text { odd }}^{(n)}=\frac{\omega_{N}+\frac{6 \lambda}{4 \cdot N} \frac{1+N_{N} R_{N}^{(n-1)}}{\omega_{N}^{2}}}{\omega_{f}+\frac{6 \lambda}{4 \cdot N} \frac{1+N_{N}}{\omega_{f}^{2}}},
$$

$$
R_{i, \mathrm{odd}}^{(n)}=\frac{\omega_{i}+\frac{6 \lambda}{4 \cdot N} \frac{2+2 N_{N-i} R_{N-i}^{(n-1)}}{\omega_{i} \omega_{N-i}}}{\omega_{f}+\frac{6 \lambda}{4 \cdot N} \frac{2+2 N_{N-i}}{\omega_{f}^{2}}}, \quad 1 \leq i \leq N-1,
$$

the n-order complexity is 


$$
D_{\left(N_{1}, \ldots, N_{N}\right)}^{(n) 2}=\left(N_{N}+1\right)\left(\ln \left(\sqrt{R_{N, \text { odd }}^{(n)}}\right)\right)^{2}+\sum_{i=1}^{N-1}\left(N_{i}+1\right)\left(\ln \left(\sqrt{R_{i, \text { odd }}^{(n)}}\right)\right)^{2}
$$

where $R_{i}^{(0)}$ is defined in (3.29).

(ii) Odd $\frac{N}{2}$ : These cases have two circles located at $\frac{N}{2}$ and $N$, pairing with each other (Fig. 4). The potential is

$$
V_{\mathrm{odd} \frac{N}{2}}^{\text {circle }}=\frac{6 \lambda}{4 \cdot N}\left[\left(\frac{1+N_{\frac{N}{2}}}{\omega_{\frac{N}{2}}^{2}}+\frac{2+2 N_{N}}{\omega_{\frac{N}{2}} \omega_{N}}\right) a_{\frac{N}{2}}^{\dagger} a_{\frac{N}{2}}+\left(\frac{1+N_{N}}{\omega_{N}^{2}}+\frac{2+2 N_{\frac{N}{2}}}{\omega_{\frac{N}{2}} \omega_{N}}\right) a_{N}^{\dagger} a_{N}\right] .
$$

The remaining contributions are those from pure "pairing" sites. Recalling Fig. 2 and the relation (4.13), we can evaluate the corresponding potential. The result is

$$
V_{\mathrm{odd} \frac{N}{2}}^{\mathrm{pair}}=\frac{6 \lambda}{4 \cdot N}\left[\sum_{i=1}^{\frac{N}{2}-1}\left(\frac{2+2 N_{N-i}}{\omega_{i} \omega_{N-i}}+\frac{2+2 N_{\frac{N}{2}-i}}{\omega_{i} \omega_{\frac{N}{2}-i}}\right) a_{i}^{\dagger} a_{i} \sum_{i=\frac{N}{2}+1}^{N-1}\left(\frac{2+2 N_{N-i}}{\omega_{i} \omega_{N-i}}+\frac{2+2 N_{\frac{3 N}{2}-i}}{\omega_{i} \omega_{\frac{3 N}{2}-i}}\right) a_{i}^{\dagger} a_{i}\right] .
$$

By adding the kinematic term (4.5) and defining the recursion relations

$$
\begin{aligned}
& R_{\frac{N}{2}, \text { even }}^{(n)}=\frac{\omega_{\frac{N}{2}}+\frac{6 \lambda}{4 \cdot N}\left(\frac{1+N_{\frac{N}{2}} R_{\frac{N}{2}}^{(n-1)}}{\omega_{\frac{N}{2}}^{2}}+\frac{2+2 N_{N} R_{N}^{(n-1)}}{\omega_{\frac{N}{2}} \omega_{N}}\right)}{\omega_{f}+\frac{6 \lambda}{4 \cdot N} \frac{1+N_{\frac{N}{2}}}{\omega_{0}^{2}}} \\
& R_{N, \text { even }}^{(n)}=\frac{\omega_{N}+\frac{6 \lambda}{4 \cdot N}\left(\frac{1+N_{N} R_{N}^{(n-1)}}{\omega_{N}^{2}}+\frac{2+2 N_{\frac{N}{2}} R_{\frac{N}{2}}^{(n-1)}}{\omega_{\frac{N}{2}} \omega_{N}}\right)}{\omega_{f}+\frac{6 \lambda}{4 \cdot N} \frac{1+N_{N}}{\omega_{0}^{2}}} \\
& R_{i, \text { even }}^{(n)}=\frac{\omega_{i}+\frac{6 \lambda}{4 \cdot N}\left(\frac{2+2 N_{N-i} R_{N-i}^{(n-1)}}{\omega_{i} \omega_{N-i}}+\frac{2+2 N_{\frac{N}{2}-i} R_{\frac{N}{2}-i}^{(n-1)}}{\omega_{i} \omega_{\frac{N}{2}-i}}\right)}{\omega_{f}+\frac{6 \lambda}{4 \cdot N} \frac{2+2 N_{N-i}}{\omega_{0}^{2}}}, \quad 1 \leq i \leq \frac{N}{2}-1,
\end{aligned}
$$

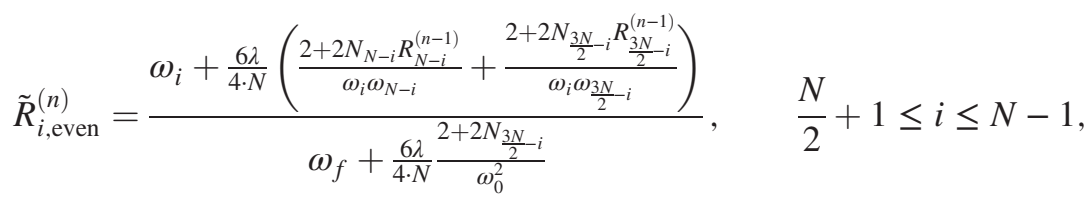

the $n$-order complexity is

$$
\begin{aligned}
D_{\left(N_{1}, \ldots, N_{N}\right)}^{(n) 2}= & \left(N_{\frac{N}{2}}+1\right)\left(\ln \left(\sqrt{R_{\frac{N}{2}, \text { even }}^{(n)}}\right)\right)^{2}+\left(N_{N}+1\right)\left(\ln \left(\sqrt{R_{N, \text { even }}^{(n)}}\right)\right)^{2} \\
& +\sum_{i=1}^{\frac{N}{2}-1}\left(N_{i}+1\right)\left(\ln \left(\sqrt{R_{i, \text { even }}^{(n)}}\right)\right)^{2}+\sum_{i=\frac{N}{2}+1}^{N-1}\left(N_{i}+1\right)\left(\ln \left(\sqrt{\tilde{R}_{i, \text { even }}^{(n)}}\right)\right)^{2},
\end{aligned}
$$

where $R_{i}^{(0)}$ is defined in (3.29).

(iii) Even $\frac{N}{2}$ : These cases have two circles locate at $\frac{N}{2}$ and $N$, pairing with each other, and two circles locate at $\frac{N}{4}$ and $\frac{3 N}{4}$, pairing with each other as well (Fig. 5). The potential is 


$$
\begin{aligned}
V_{\text {even } \frac{N}{2}}^{\text {circle }}= & \frac{6 \lambda}{4 \cdot N}\left[\left(\frac{1+N_{\frac{N}{2}}}{\omega_{\frac{N}{2}}^{2}}+\frac{2+2 N_{N}}{\omega_{\frac{N}{2}} \omega_{N}}\right) a_{\frac{N}{2}}^{\dagger} a_{\frac{N}{2}}+\left(\frac{1+N_{N}}{\omega_{N}^{2}}+\frac{2+2 N_{\frac{N}{2}}}{\omega_{\frac{N}{2}} \omega_{N}}\right) a_{N}^{\dagger} a_{N}\right. \\
& \left.+\left(\frac{1+N_{\frac{N}{4}}}{\omega_{\frac{N}{4}}^{2}}+\frac{2+2 N_{\frac{3 N}{4}}}{\omega_{\frac{N}{4}} \omega_{\frac{3 N}{4}}}\right) a_{\frac{N}{4}}^{\dagger} a_{\frac{N}{4}}+\left(\frac{1+N_{\frac{3 N}{4}}}{\omega_{\frac{3 N}{4}}^{2}}+\frac{2+2 N_{\frac{N}{4}}}{\omega_{\frac{3 N}{4}} \omega_{\frac{N}{4}}}\right) a_{\frac{N}{4}}^{\dagger} a_{\frac{3 N}{4}}\right] .
\end{aligned}
$$

Again, the remaining contributions are those from pure pairing sites. We find

$$
V_{\text {even } \frac{N}{2}}^{\text {pair }}=\frac{6 \lambda}{4 \cdot N}\left[\sum_{i=1, \neq \frac{N}{4}}^{\frac{N}{2}-1}\left(\frac{2+2 N_{N-i}}{\omega_{i} \omega_{N-i}}+\frac{2+2 N_{\frac{N}{2}-i}}{\omega_{i} \omega_{\frac{N}{2}-i}}\right) a_{i}^{\dagger} a_{i} \sum_{i=\frac{N}{2}+1, \neq \frac{3 N}{4}}^{N-1}\left(\frac{2+2 N_{N-i}}{\omega_{i} \omega_{N-i}}+\frac{2+2 N_{\frac{3 N}{2}-i}}{\omega_{i} \omega_{\frac{3 N}{2}-i}}\right) a_{i}^{\dagger} a_{i}\right] .
$$

The above result is the same as the odd $\frac{N}{2}$, i.e., (5.7), but drop the circle at $\frac{N}{4}$ and $\frac{3 N}{4}$ since the potential of the two circles has been considered in $V_{\text {even } \frac{N}{2}}^{\text {circle }}$.

By adding the kinematic term (4.5) and defining the recursion relations

$$
\begin{aligned}
& R_{\frac{N}{4}, \text { even }}^{(n)}=\frac{\omega_{\frac{N}{4}}+\frac{6 \lambda}{4 \cdot N}\left(\frac{1+N_{\frac{N}{4}} R_{\frac{N}{4}}^{(n-1)}}{\omega_{\frac{N}{4}}^{2}}+\frac{2+2 N_{\frac{3 N}{4}} R_{\frac{3 N}{4}}^{(n-1)}}{\omega_{\frac{N}{4}} \frac{3 N}{4}}\right)}{\omega_{f}+\frac{6 \lambda}{4 \cdot N} \frac{1+N_{\frac{N}{4}}}{\omega_{0}^{2}}}, \\
& R_{\frac{3 N}{4}, \text { even }}^{(n)}=\frac{\omega_{\frac{3 N}{4}}+\frac{6 \lambda}{4 \cdot N}\left(\frac{1+N_{\frac{3 N}{4}} R_{\frac{3 N}{4}}^{(n-1)}}{\omega_{\frac{3 N}{4}}^{2}}+\frac{2+2 N_{\frac{N}{4}} R_{\frac{N}{4}}^{(n-1)}}{\omega_{\frac{N}{4}} \omega_{\frac{3 N}{4}}}\right)}{\omega_{f}+\frac{6 \lambda}{4 \cdot N} \frac{2+2 N_{\frac{N}{4}}}{\omega_{0}^{2}}},
\end{aligned}
$$

the n-order complexity is

$$
\begin{aligned}
D_{\left(N_{1}, \ldots, N_{N}\right)}^{(n) 2}= & \left(N_{\frac{N}{2}}+1\right)\left(\ln \left(\sqrt{R_{\frac{N}{2}, \text { even }}^{(n)}}\right)\right)^{2}+\left(N_{N}+1\right)\left(\ln \left(\sqrt{R_{N, \text { even }}^{(n)}}\right)\right)^{2} \\
& +\left(N_{\frac{N}{4}}+1\right)\left(\ln \left(\sqrt{R_{\frac{N}{4}, \text { even }}^{(n)}}\right)\right)^{2}+\left(N_{\frac{3 N}{4}}+1\right)\left(\ln \left(\sqrt{R_{\frac{3 N}{4}, \text { even }}^{(n)}}\right)\right)^{2} \\
& +\sum_{i=1, \neq \frac{N}{4}}^{\frac{N}{2}-1}\left(N_{i}+1\right)\left(\ln \left(\sqrt{R_{i, \text { even }}^{(n)}}\right)\right)^{2}+\sum_{i=\frac{N}{2}+1, \neq \frac{3 N}{4}}^{N-1}\left(N_{i}+1\right)\left(\ln \left(\sqrt{\tilde{R}_{i, \text { even }}^{(n)}}\right)\right)^{2} .
\end{aligned}
$$

These general formulas allow one to obtain higher-order complexity for excited states at any $N$ coupled oscillators, which is a lattice version of $\lambda \phi^{4}$ theory.

While the formulas we used involve the bare parameters to extract physics, we have to rewrite the expressions above in terms of the renormalized quantities. The issues have been studied in [17] and we summarize it below. First, following [19], the mass has relation

$$
(m \delta)^{2}=\left(m_{R} \delta\right)^{2}-\frac{\lambda_{R} \delta^{4-d}}{2} I\left(m_{R} \delta\right)+O\left(\lambda_{R}^{2}\right)
$$

where $m_{R}$ is the renormalized mass and $\lambda_{R}$ is the renormalized coupling defined at zero momentum. A running renormalized coupling can also be defined at finite momentum $\mu$ and for the leading order in the coupling, one can replace $\lambda_{R}$ by $\lambda_{R}(\mu)$. Here,

$$
I\left(m_{R} \delta\right)=\prod_{i=1}^{d}\left[\int_{-\pi}^{\pi} \frac{d l_{i}}{(2 \pi)}\right] \frac{1}{\left(m_{R} \delta\right)^{2}+4 \sum_{i=1}^{d} \sin ^{2}\left(\frac{l_{i}}{2}\right)}
$$


For $d=2$,

$$
(m \delta)^{2}=\left(m_{R} \delta\right)^{2}-\frac{\lambda_{R} \delta^{2}}{2}\left[C_{0}-2 C_{1} \log \left(m_{R} \delta\right)-C_{2}\left(m_{R} \delta\right)^{2}+\frac{1}{32 \pi}\left(m_{R} \delta\right)^{2} \log \left(\left(m_{R} \delta\right)^{2}\right)+\mathcal{O}\left(\left(m_{R} \delta\right)^{4}\right)\right]
$$

Here, $C_{0}=0.28, C_{1}=0.08$, and $C_{2}=0.02$. For $d \geq 3$,

$$
(m \delta)^{2}=\left(m_{R} \delta\right)^{2}-\frac{\lambda_{R} \delta^{4-d}}{2}\left[C_{0}-C_{2}\left(m_{R} \delta\right)^{2}+\left.\frac{1}{16 \pi^{2}}\left(m_{R} \delta\right)^{2} \log \left(\left(m_{R} \delta\right)^{2}\right)\right|_{d=4}+\mathcal{O}\left(\left(m_{R} \delta\right)^{4}\right)\right]
$$

in which for $d=4$ there is an extra log term. The values of $C_{0}$ and $C_{2}$ for various dimensions can be found in [17]. Note that in leading order $\hat{\lambda}_{0}=\lambda_{R}$, where $\lambda_{R}$ is the renormalized coupling.

\section{B. Complexity of $N$ coupled oscillators: Numerical results}

We now use the above formulas to perform numerical calculations and plot several diagrams to illuminate the properties of complexity.

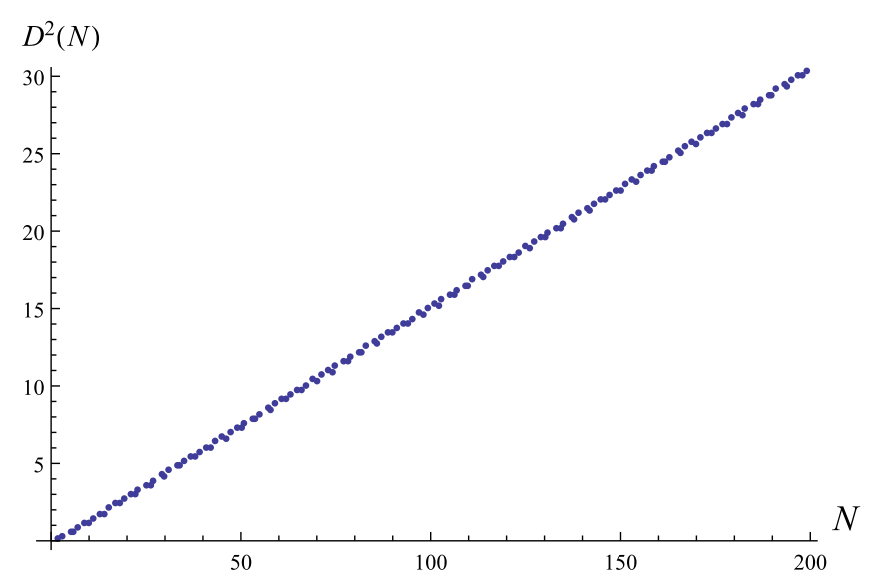

FIG. 13. Complexity vs lattice site number $N$.

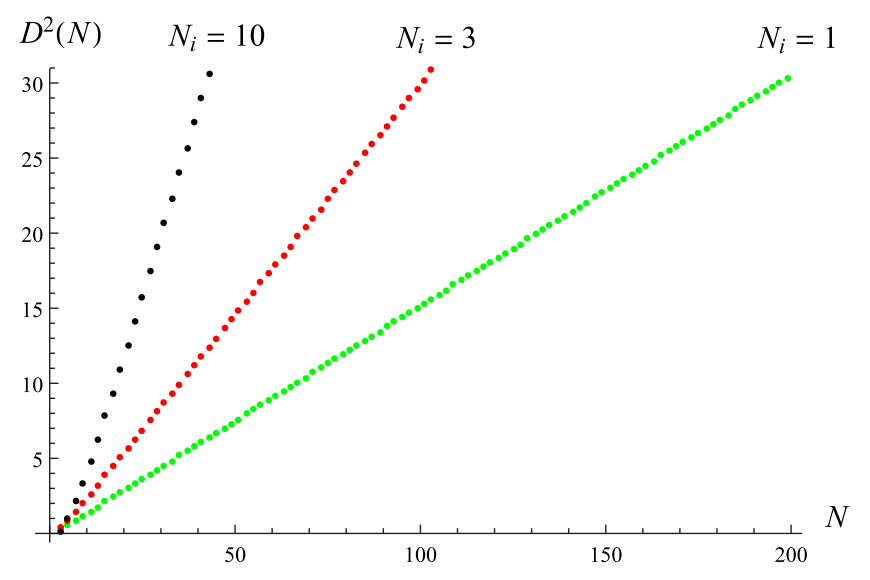

FIG. 14. Complexity vs excited state $N_{i}$.
(1) We plot in Fig. 13 the complexity for various lattice site number $N$.

It shows that the complexity increases with site number $N$, as that in free theory. This is consistent with the relation CV conjecture [8], since in one dimension the volume is proportional to site number $N$. To plot Fig. 13 (and following figures), we choose the scale of $\omega=1$ and use the following values: $N_{i}=1, \omega_{f}=1$, and $\lambda=0.1$. The dependence of complexity on $N_{i}, \omega_{f}$, or $\lambda$ is illuminated in the following figures.

(2) We plot in Fig. 14 the complexity for various excited states $N_{i}$.

It shows that the complexity becomes larger in higher excited states, as that in free theory.

(3) We plot in Fig. 15 the complexity for various reference-state frequencies $\omega_{f}$.

It shows that the complexity becomes larger for large $\omega_{f}$, as that in free theory.

(4) We plot in Fig. 16 the complexity for various coupling constants $\lambda$ in lattice $\lambda \phi^{4}$ theory.

It shows that the complexity may increase or decrease while increasing coupling constant $\lambda$. The property of how the complexity depends on $\lambda$ can be seen, for example, from Eqs. (3.36) and (3.37). The interaction correction to complexity in the two 

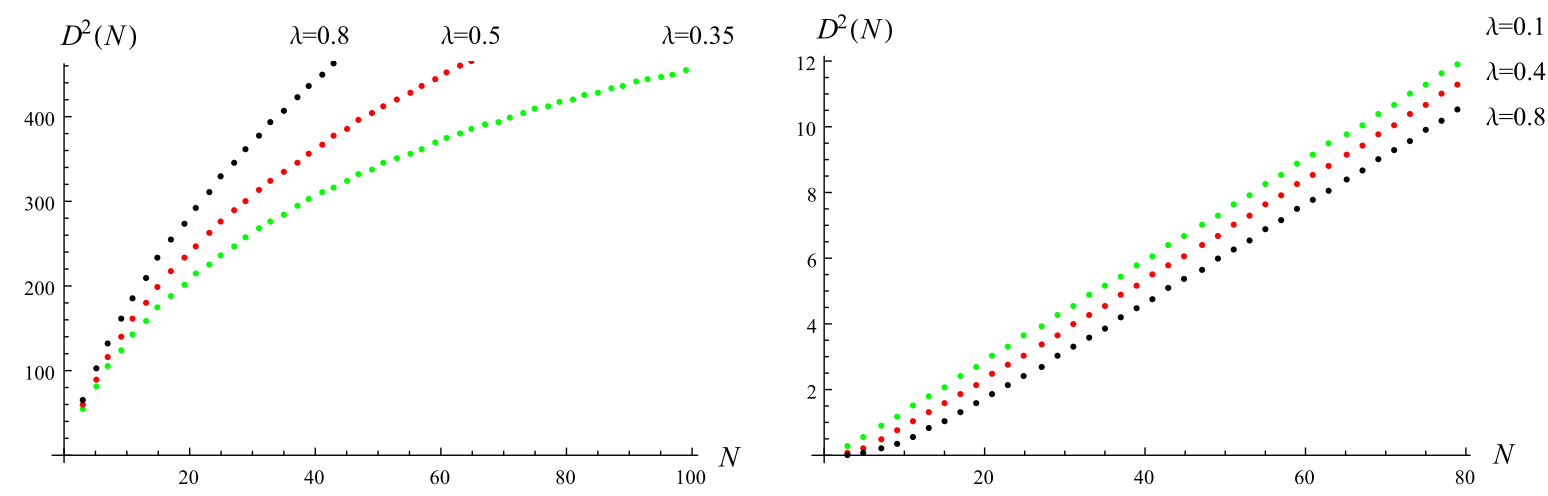

FIG. 16. Complexity vs coupling constant $\lambda$. Left: $\omega=10, \omega_{f}=0.01$. Right: $\omega=\omega_{f}=1$.

relations is proportional to the coefficient of $\frac{3 \lambda}{2 \omega_{f}}$, which is negative for large $\omega_{f}$ and becomes positive for small $\omega_{f}$. Figure 16 is consistent with this argument.

\section{CONCLUDING REMARKS}

We adopt the operator approach to compute the complexity of the lattice $\lambda \phi^{4}$ scalar theory. A perturbation algorithm has been developed for computing the complexity to obtain the general formulas (5.5), (5.12), and (5.17), which can be used to obtain higher-order complexity of excited states for any $N$ lattice sites. The interaction correction to complexity may be positive or negative depending on the magnitude of reference-state frequency.

We conclude the paper by remarking that our algorithm is based on a simple relation

$$
\lambda a_{j}^{\dagger} a_{j} a_{j}^{\dagger} a_{j} \rightarrow \lambda N_{j} a_{j}^{\dagger} a_{j} \rightarrow \lambda N_{j} R_{j}^{(n-1)} a_{j}^{\dagger} a_{j},
$$

in which the first arrow is due to the perturbation property, while the second one is used to calculate the complexity. The relation is explained in Sec. III. B. The similar relation could be found in many other systems, as shown in the following, for example.

(i) It is easy to see that our method could be used in interacting Fermion theory. (ii) For the theory that has two different field operators $a_{j}$ and $b_{j}$ and the associated interaction is $\lambda \phi^{2} \xi^{2}$, the relation will become

$$
\begin{aligned}
\lambda a_{j}^{\dagger} a_{j} b_{j}^{\dagger} b_{j} \rightarrow & \frac{\lambda}{2} N_{j}^{(b)} a_{j}^{\dagger} a_{j}+\frac{\lambda}{2} N_{j}^{(a)} b_{j}^{\dagger} b_{j} \\
\rightarrow & \frac{\lambda}{2} N_{j}^{(b)} R_{j}^{(b)(n-1)} a_{j}^{\dagger} a_{j} \\
& +\frac{\lambda}{2} N_{j}^{(a)} R_{j}^{(a)(n-1)} b_{j}^{\dagger} b_{j},
\end{aligned}
$$

in which the fields $\phi$ and $\xi$ could be boson or Fermion fields.

(iii) For the $\lambda \phi^{6}$ theory, the relation will become

$$
\lambda a_{j}^{\dagger} a_{j} a_{j}^{\dagger} a_{j} a_{j}^{\dagger} a_{j} \rightarrow \lambda\left(N_{j}\right)^{2} a_{j}^{\dagger} a_{j} \rightarrow \lambda\left(N_{j} R_{j}^{(n-1)}\right)^{2} a_{j}^{\dagger} a_{j} .
$$

Of course, the associated diagrams and basic rules in each case shall be slightly modified.

In this way, our algorithm can be applied to many quantum field theories and several many-body models in condensed matter. We will study the problem in the next series of papers.
[1] M. van Raamsdonk, Building up spacetime with quantum entanglement, Gen. Relativ. Gravit. 42, 2323 (2010).

[2] B. Swingle and M. Van Raamsdonk, Universality of gravity from entanglement, arXiv:1405.2933.

[3] N. Lashkari, M. B. McDermott, and M. Van Raamsdonk, Gravitational dynamics from entanglement thermodynamics, J. High Energy Phys. 04 (2014) 195.
[4] T. Faulkner, M. Guica, T. Hartman, R. C. Myers, and M. Van Raamsdonk, Gravitation from entanglement in holographic CFTs, J. High Energy Phys. 03 (2014) 051.

[5] J. Maldacena, Eternal black holes in anti-de Sitter, J. High Energy Phys. 04 (2003) 021.

[6] T. Hartman and J. Maldacena, Time evolution of entanglement entropy from black hole interiors, J. High Energy Phys. 05 (2013) 014. 
[7] J. Maldacena and L. Susskind, Cool horizons for entangled black holes, Fortschr. Phys. 61, 781 (2013).

[8] L. Susskind, Computational complexity and black hole horizons, Fortschr. Phys. 64, 24 (2016).

[9] D. Carmi, S. Chapman, H. Marrochio, R. C. Myers, and S. Sugishita, On the time dependence of holographic complexity, J. High Energy Phys. 11 (2017) 188.

[10] A. R. Brown, D. A. Roberts, L. Susskind, B. Swingle, and Y. Zhao, Holographic Complexity Equals Bulk Action?, Phys. Rev. Lett. 116, 191301 (2016).

[11] A. R. Brown, D. A. Roberts, L. Susskind, B. Swingle, and Y. Zhao, Complexity, action, and black holes, Phys. Rev. D 93, 086006 (2016).

[12] S. Chapman, H. Marrochio, and R. C. Myers, Complexity of formation in holography, J. High Energy Phys. 01 (2017) 62.

[13] R. A. Jefferson and R. C. Myers, Circuit complexity in quantum field theory, J. High Energy Phys. 10 (2017) 107.
[14] S. Chapman, M. P. Heller, H. Marrochio, and F. Pastawski, Towards Complexity for Quantum Field Theory States, Phys. Rev. Lett. 120, 121602 (2018).

[15] R. Khan, C. Krishnan, and S. Sharma, Circuit complexity in fermionic field theory, Phys. Rev. D 98, 126001 (2018).

[16] L. Hackl and R. C. Myers, Circuit complexity for free fermions, J. High Energy Phys. 07 (2018) 139.

[17] A. Bhattacharyya, A. Shekar, and A. Sinha, Circuit complexity in interacting QFTs and RG flows, J. High Energy Phys. 10 (2018) 140.

[18] W.-H. Huang, Operator approach to complexity: Excited states, Phys. Rev. D 100, 066013 (2019).

[19] J. Smit, Introduction to Quantum Fields on a Lattice, Cambridge Lecture Notes in Physics (Cambridge University Press, Cambridge, England, 2002), https://www.cambridge .org/core/books/introduction-to-quantum-fields-on-alattice/8573D89B238 102666F0B9D5F7FB3ED12. 